\title{
Las agencias de relaciones públicas y su influencia en el periodismo de empresas y negocios en Chile
}

\section{Public Relation agencies and their influence on business journalism in Chile}

\author{
Mario Álvarez \\ Periodista, Universidad de Chile \\ alvarezfuentes@gmail.com
}

\begin{abstract}
Resumen
Este artículo aborda la influencia de las agencias de relaciones públicas en el quehacer cotidiano del periodismo de empresas y negocios en Chile y se basa en la experiencia profesional adquirida durante diez años como reportero de este sector para diferentes medios nacionales.

El texto propone que las agencias de relaciones públicas son fruto de un contexto de consolidación histórica del neoliberalismo en Chile. Se destaca su importancia en la construcción de poder simbólico de los grupos de interés, principalmente para las empresas que pueden pagar por sus servicios. A poyándose en el concepto de subsidios de información intenta proveer una perspectiva para aproximarse a los "favores" que estas agencias ofrecen a los periodistas, a través de diversas estrategias tendientes a mejorar la cobertura de los clientes (como los comunicados de prensa, los viajes organizados para la prensa) y su impacto en la cobertura de noticias. Finalmente, se enfatiza la necesidad de pasar de una visión sobre la relación de las relaciones públicas y el periodismo como parte de una alianza estratégica que incluya sus consideraciones y dimensiones éticas.
\end{abstract}

Palabras claves: Relaciones públicas, agencias, comunicaciones, periodismo, empresas, negocios.

\footnotetext{
Abstract

This article addresses the influence of PR agencies on the day-by-day work of business and financial journalism based on professional experience working as a journalist on business affairs for over ten years at different national newspapers.

The text considers PR agencies as a result of a scenario of historical consolidation of neo liberal ideology in Chile. It draws attention to their importance in the construction of
} 
symbolic power of interest groups, mainly economic power of companies that can pay for their services. By using the concept of information subsidies, it moves to provide an overview of the so-called "bribes" these agencies offer journalists. It also looks at presstravels organised by these agencies and their impact on news coverage. Finally, it emphasises the need to move away from the examination of the relationship between public relations and journalism as part of strategic alliance to include ethical considerations/dimensions.

Key words: Public relations, PR, agencies, communications, journalism, companies, business.

\section{Introducción}

El modelo hegemónico de financiamiento de la prensa es el comercial, donde los medios captan audiencias y lectores (atractivos en volumen y en características de consumo) para ofrecerlos a los avisadores. Eso permite financiar la planilla profesional, técnica y ejecutiva, la impresión y distribución, entre otros gastos recurrentes de un diario o una revista. A eso se suman algunos pesos que entran por la vía de la venta en kioscos y suscripciones, los que son menores en comparación a la relevancia de los ingresos por la vía de la publicidad. Al revisar los ingresos de los principales diarios nacionales, se puede estimar que para El Mercurio y La Tercera, el avisaje representa un 85 por ciento de su facturación, mientras que para Las Últimas Noticias la proporción es de 77 por ciento y en La Cuarta, 68 por ciento' ${ }^{1}$.

Del mismo modo, en las últimas dos décadas, Chile ha experimentado un crecimiento económico sin precedentes y se instaló una competencia entre los dos principales diarios orientados al segmento socioeconómico ABC1. La Tercera ha ganado terreno frente a El Mercurio en la captación de inversión publicitaria en los últimos diez años. Si en 2001 el diario de Álvaro Saieh concentraba el 12 por ciento de la inversión en los principales diarios nacionales, en 2011 esa cuota subió a 22 por ciento, mientras que la del diario de Agustín Edwards bajó de 52 por ciento a 37 por ciento de una torta que se ha más que duplicado en el mismo período ${ }^{2}$.

Estos dos procesos se han dado tras el fin de la dictadura de Augusto Pinochet, cuya expresión económica fue la instalación de un modelo neoliberal que acentúa la concentración de la propiedad, la participación en mercados y en ventas (o captación de audiencias, en el caso de los medios). En los años 90, durante la transición a la democracia, los grupos privilegiados con este orden heredado buscan salvaguardarlo y, entre otras herramientas, las secciones y medios de economía, empresas y negocios adquieren mayor relevancia.

\footnotetext{
1 Según datos del proyecto PLU1104 Publicidad privada y su influencia en el ejercicio del periodismo y la libertad de expresión.

2 Según datos de inversión publicitaría de Megatime 2001-2011, ajustados.
} 
El siguiente no es un artículo basado en una investigación sistemática de largo aliento en la materia. No es más, pero tampoco menos, que un testimonio que articula la experiencia de diez años cubriendo economía -y, en particular, empresas- para diferentes medios de cobertura nacional y que se ilumina con la contribución de investigaciones sobre el quehacer de las agencias de relaciones públicas desarrolladas en distintos países.

Se propone una reflexión profesional sobre la forma que adquiere el trabajo de los reporteros bajo este esquema de financiamiento y competencia entre los diarios y en un clima de consolidación histórica del proyecto neoliberal. El foco se pone en las agencias de relaciones públicas que trabajan para las grandes empresas, cuyo rol será analizado en consecuencia con estos contextos.

La primera parte, y a modo de introducción, se incluye un relato testimonial de la relación de los periodistas con la censura comercial durante su práctica cotidiana, luego se desarrolla una observación, descripción y conceptualización del quehacer de las agencias de relaciones públicas y, finalmente, proponemos una reflexión sobre los desafíos éticos planteados por los escenarios abordados.

\section{Mitos y leyendas de la censura comercial en la prensa chilena}

Lo que convoca esta reflexión son los resultados del estudio "Publicidad privada y sus implicancias para el ejercicio del periodismo y las libertades de expresión y prensa en Chile”, cuyas principales conclusiones son publicados en esta misma edición. En él se examina la cobertura dada por diarios nacionales y regionales a las noticias relacionadas con la colusión de precios cometida por las tres principales cadenas de farmacias de Chile (Cruz Verde, SalcoBrand y Ahumada) buscando dilucidar si la prensa chilena es ajena -o no-a restricciones directas o indirectas de sus avisadores.

Como una de sus principales conclusiones, los investigadores observan que no es posible advertir una relación directa entre avisadores y un "tipo" de cobertura que podría haber estado contaminada por las presiones económicas de la publicidad. No se puede presumir que los anunciantes, en este caso, determinaron el contenido publicado en una dirección que los favoreciera.

Tales observaciones son coincidentes con la experiencia cotidiana de quienes trabajan en este sector. Allí, la censura comercial, entendida como eliminación o alteración de contenidos desde las páginas de un diario ya editado producto de presiones ejercidas por avisadores, se vive más como un relato compartido que como una práctica concreta.

3 Claudia Lagos, Laureano Checa, Cristián Cabalin y Macarena Peña y Lillo (PLU1104). 
Cualquier reportero de un medio de comunicación de alcance nacional, no importa el frente noticioso que cubra, tiene dos grandes grupos de referencia (y, por lo tanto, de socialización): el primero, sus compañeros de redacción; y el segundo, los colegas de frente noticioso con los que comparte largas horas de espera y reporteo en terreno que permiten cultivar lazos de colaboración, a la vez que de competencia.

En ambos contextos se comparten historias orales que se traspasan de generación en generación -cual mito- sobre artículos que estaban diagramados al cierre de la edición, pero que al día siguiente no aparecieron publicados por llamados de los avisadores haciendo ver su inconveniencia y pidiendo su retiro. A pesar de las contradicciones o ciertos vacíos que pueden advertirse en estas historias, estas van conformando y alimentando un gran relato sobre la presión con que supuestamente debe lidiar el periodista al reportear y escribir sobre empresas. La censura comercial es algo que a alguien le ocurrió; historias de oídas, de las cuales es muy difícil encontrar y estar cara a cara con sus protagonistas o hallar pistas o pruebas. Y cuando finalmente estas historias se verifican, parecen perder dramatismo y entenderse como parte de la rutina y de las reglas del juego implícitas del ejercicio profesional del periodismo económico.

En la sala de redacción, además, el discurso de editores, directores y gerentes es que hay libertad para trabajar y publicar, que el único límite es el mal reporteo, los datos no verificados o las fuentes no contrastadas, pero que una vez observados estos aspectos, una nota debiera salir publicada, aun a disgusto de los avisadores.

$\mathrm{Al}$ menos desde la experiencia, lo cierto es que la censura comercial directa es mucho menos evidente y, además, menos frecuente de lo que podría intuirse desde fuera de las salas de redacción, y no afecta severamente la estabilidad laboral de los periodistas que cubren el sector. $\mathrm{Al}$ menos en prensa. La experiencia profesional muestra que los reporteros que han vivido casos concretos de censura comercial son una minoría entre quienes reportean en secciones de economía y negocios.

Sin embargo, es posible afirmar que el poder de los avisadores para controlar los contenidos en los medios -si bien no es absoluto- es importante: puede que no signifique el despido o la caída en desgracia de los reporteros más agudos; o que no eliminen contenidos de manera descarada. Tampoco significa que siempre los avisadores retiran publicidad como castigo a contenidos que consideren críticos a sus marcas o empresas. Pero sí es posible advertir ediciones mañosas de los textos, manipulación en los sentidos de los titulares, recorte o restricciones para visibilizar a ciertas fuentes $-\mathrm{y}$, por lo tanto, a ciertas perspectivas de mundo- o la omisión de datos específicos.

Finalmente, cuando la censura comercial de la que tanto se había escuchado ocurre en realidad en una nota propia, los editores, en vez de reprender al reportero, lo acogen, lo cuidan y se lo trata como a alguien que aprendió una dura, pero valiosa lección para su futuro profesional.

La suma del contexto general en que se reportea, las relaciones que se establecen con las fuentes y sus intermediarios y la precariedad general en que se ejerce periodismo en Chile, importan más que la censura para dibujar los márgenes en que se mueven los reporteros en 
cualquier sección. Lo que hace especial a la cobertura de negocios y empresas no es la censura comercial sino las historias sobre ella. Ese relato tiene una mecánica y unos agentes particulares que no aparecen en otros ámbitos del periodismo (crónica, cultura, deportes o política, por ejemplo) y se agrega como otro instrumento normalizador con la función moral de establecer lo que se debe y no debe hacer. Uno de dichos actores son las agencias de comunicaciones, consultoras comunicacionales o empresas de relaciones públicas.

\section{Censura: cara e ineficiente}

Creer que el poder económico no controla los contenidos de la prensa solo porque la censura comercial es poco frecuente es ingenuo: sí lo hacen, pero no usan una herramienta tan costosa, es decir, cara e ineficiente (adjetivos proscritos en el mundo de los negocios). Por propiciar la censura, una empresa arriesga pagar con la mala disposición de editores y reporteros hacia ella y de los demás periodistas del frente y, a cambio, no se asegura que esa noticia no sea conocida por el público a través de otros medios.

Así, en vez de optar por la censura, las empresas han desarrollado recursos que permiten que, antes siquiera de que un hecho que los afecte pueda ser publicado, este ni siquiera entre en pauta. No alcanza a ser noticia. Las probabilidades de que un conflicto laboral, un abuso a los consumidores o un atentando a la salud o al medio ambiente se transformen en noticia en una sección de economía y negocios, en los medios de cobertura nacional, son escasas: ni siquiera llegan a la reunión de pauta (Sunkel et al., 2003). No están en la agenda de los reporteros. No alcanzan a ser discutido. Por lo tanto, no hay un contenido conflictivo que eventualmente signifique que un avisador quiera que no se publique y presione en ese sentido.

Una de los recursos de dicho poder es la profesionalización de sus estrategias comunicacionales, a través de intermediaros: agencias de relaciones públicas. Estas organizaciones despliegan una serie de estrategias para construir y consolidar relaciones con los periodistas del área, en particular, así como con los medios de comunicación -y sus planas ejecutivas- que inhiben -e incluso condenan- la crítica. Es decir, contribuyen a desincentivar el rol fiscalizador o de cuarto poder que se le asigna al periodismo en una sociedad democrática. Estrategias que regulan las relaciones con los periodistas y los medios, de tal suerte que disminuyen las posibilidades de recurrir al método más sucio y doloroso de controlar un contenido: la censura.

\section{Las agencias de comunicaciones como engranaje del discurso neoliberal}

Las agencias de relaciones públicas son el fruto de condiciones políticas y económicas concretas que han permitido su auge en distintas partes del mundo y que se replican en Chile con características bastantes similares. 
A la luz de los estudios de Jacquie L'Etang (2004), Lee Edwards (2012) trazó el origen de las formas modernas de relaciones públicas en el Reino Unido hacia fines del siglo XIX y comienzos del XX, como parte de los esfuerzos por expandir y sustentar al Imperio Británico. Uno de los ejemplos es la política de fomento a la producción y distribución de películas durante los años 30 del siglo XX para promover entre sus colonias un modelo de entendimiento que favoreciera su expansión. Durante la desintegración del Imperio Británico, las relaciones públicas se desplegaron como herramienta para que las colonias vivieran una transición a la independencia sin mayores sobresaltos. En ese volátil contexto histórico es que las organizaciones comerciales -que habían sido punta de lanza del Imperio en la expansión territorial- comenzaron a diseñar y desplegar campañas de comunicación para proteger sus intereses en las convulsionadas colonias.

Desde la Segunda Guerra Mundial, las relaciones públicas en Europa pasaron desde una función unidimensional de agencias de prensa a ser una sofisticada red de comunicación que conecta los elementos más poderosos de nuestra sociedad (Turow, 1989). A través de ella, empresas, sindicatos, gobiernos u organizaciones no gubernamentales y otros grupos de interés intentan capturar a los variados públicos con los que debe lidiar: consumidores, empleados, inversionistas y reguladores, entre otros.

En el mundo privado, las relaciones públicas tomaron su forma actual de agencias profesionalizadas con el advenimiento de las desregulaciones profesionales y las privatizaciones de empresas desarrolladas durante la década de los 80 y los 90 . El primer proceso abrió nuevos y lucrativos mercados para ayudar a profesionales como abogados o consultores a comunicarse con las audiencias, mientras que para las segundas, cumplieron la misión fundamental de promover las nuevas compañías privadas y crear en la sociedad una mentalidad proclive a invertir en ellas.

Esta expansión de las relaciones públicas corporativas coincidió con un fuerte cambio político hacia el libre mercado en la formulación de políticas públicas que benefició al capital por sobre el trabajo. Tales cambios fueron posibles gracias al apoyo de medios de comunicación conservadores, lo que Davies (2000) ha denominado el control corporativo de los medios de comunicación, y que se ha perfeccionado gracias a un uso más intensivo de las relaciones públicas.

El contexto histórico antes descrito coincide con el que permitió también el florecimiento de las primeras agencias formales de comunicación en Chile: HK Strategies, Extend, Tironi y Asociados o Burson Marsteller Chile surgieron en los año 90 y se mantienen vigentes.

Las agencias aparecen en un ambiente de consolidación histórica del neoliberalismo como orden social y económico y, en un ambiente democrático, permiten legitimar un modelo impulsado por la dictadura de Augusto Pinochet (1973-1990). Los años 90 fueron los de la validación de operaciones que se habían impuesto por la fuerza durante las dos décadas anteriores tales como la obligatoriedad de las cotizaciones previsionales a empresas privadas y de seguro de salud, la privatización de empresas públicas y servicios básicos, la precarización laboral y la preponderancia de los equilibrios macroeconómicos, entre otras. (Larraín, 2005). 
Otra relación de las agencias de relaciones públicas y el neoliberalismo es que estas son fruto del vasto concepto de externalización, que consiste en la delegación de actividades que no son claves a una entidad externa que esté especializada en dichas operaciones (Bravard, 2007). En este marco, una minera debiera dedicarse a extraer y comerciar minerales y dejar la alimentación de sus trabajadores a un operador de casinos, así como su relación con los medios de prensa, en manos de una agencia de relaciones públicas.

En síntesis, las relaciones públicas modernas y su desarrollo están estrechamente vinculados al poder político y al desarrollo y consolidación de un modelo neoliberal. Estas han producido discursos que permiten y mantienen un ambiente que favorece los intereses corporativos $y$ han ido más allá de la mera intención de vender productos o servicios. (Edwards, 2012).

Las agencias de relaciones públicas forman parte de un entramado que ha sustentado las ideas del neoliberalismo en el discurso público chileno. Esta red está tejida por los modelos de financiamiento de los medios de comunicación - dependientes de la inversión publicitaria de las grandes empresas-, el surgimiento de diarios especializados, la creación y ampliación de secciones de economía y negocios en diarios tradicionales y la fundación de centros de estudios orientados a influir en el debate público, dando sustento argumental a las posturas ideológicas.

\section{El comunicado de prensa y su poder simbólico}

Las agencias de relaciones públicas han adquirido un estatus relevante para la cultura, no solo por su crecimiento, su expansión o la cantidad de empresas a las que asesoran, sino también por su localización dentro del flujo de información que será publicado o emitido al aire. En palabras de Edwards (2012), no son solo bisagras o mensajeras, sino que constituyen una forma simbólicamente violenta de intermediación cultural, diseñada, en última instancia, para generar poder simbólico para grupos de intereses, centradas en que las empresas para las cuales trabajan, acrecienten y consoliden sus posiciones de poder.

En el contexto de alta competencia por participación de mercados, diferenciarse de los competidores permite a las empresas agregarles valor simbólico a los atributos, actitudes o prácticas (opiniones frente a ciertas políticas públicas, formas de consumo o modelos de negocios), que refuercen su propia posición y debiliten la de los otros actores (Edwards, 2006).

Entonces, en la medida en que no solo vale más quién haga el mejor producto u ofrezca un buen servicio, y dado que la lucha se da más bien en el campo de lo simbólico, las empresas se han visto obligadas a invertir en relaciones públicas, con tal de desplegar y fortalecer su capital económico a través de estrategias comunicacionales que compensen su intrínseca falta de capital cultural y la falta de interés del público en sus asuntos (Davies, 2000).

El crecimiento de las relaciones públicas es la contracara del dramático declive en los recursos destinados a la producción de noticias (Davies, 2000). Las noticias son cada vez menos populares para muchos lectores, televidentes y avisadores, mientras que aumenta la 
atracción por el entretenimiento. Este proceso se desarrolla en paralelo con la agudización de la competencia entre los medios de comunicación, una creciente desregulación gubernamental sobre esta industria y la introducción de las lógicas del libre mercado en los servicios de noticias.

Así, en la medida en que la gestión de los costos se vuelve más importante, decayeron los ideales profesionales y la voluntad para financiar investigaciones periodísticas costosas. Siguiendo a Davies, los periodistas están sometidos a una mayor presión por generar resultados, con menos recursos para trabajar. Con ello, dice Davies (2000), se han debilitado el periodismo de investigación, la calidad editorial y se ha acrecentado la dependencia de las fuentes.

La diversidad de organizaciones que hoy recurren a intermediarios para seducir a sus distintas audiencias envían mensajes públicos cuidadosamente construidos para poner a este grupo de interés, sus objetivos o productos a la mejor luz posible, de manera que sus públicos objetivos piensen y se comporten de una forma que beneficie a este grupo de interés.

La forma más común que adquieren estos mensajes son los comunicados de prensa $-\mathrm{O}$ gacetillas, como se conocen en otros países-: textos de difusión que se envían a los periodistas con el objetivo de que sean publicados. En un contexto donde la hibridez de los géneros se ha convertido en regla, estos textos son redactados en clave periodística, siguiendo las reglas básicas del periodismo informativo. Sin embargo, tal como señala Sissons (2012), el comunicado mezcla equilibradamente el valor noticioso con la promoción de tal forma que capta la atención de los periodistas, sin perder de vista su objetivo de difusión al público general.

En un solo día de cobertura normal en las redacciones, los periodistas pueden recibir una treintena de estos documentos, producidos bajo estándares similares a los que requiere la producción noticiosa en un medio: lo escribe un profesional de una agencia de relaciones públicas, lo revisa su "editor" -o jefe de cuenta-, el cual lo envía a la empresa-cliente para que su encargado de comunicaciones o algún ejecutivo pertinente dé el visto bueno para enviarlo a los medios. Qué se dice y cómo se dice es fundamental y se le dedica tiempo y procedimientos.

Este exhaustivo proceso de revisión antes de poner en circulación un comunicado, se entiende en tanto resulta un mecanismo eficiente para incidir en los contenidos de los medios. Dos estudios citados por Turow dan pistas sobre aquello: Blyskal y Blyskal (1985) demostró que el 50\% de los artículos de The Wall Street Journal se iniciaron a partir de un comunicado de prensa. Esto confirmaba los hallazgos de un trabajo de 1980 publicado en Columbia Journalism Review: de 53 artículos basados en comunicados de prensa 32 estaban copiados prácticamente de manera literal.

La falta de voluntad o la imposibilidad de los reporteros para verificar la fuente de los comunicados y, por lo tanto, la veracidad de su contenido, impide que el lector infiera el origen de la información y su real valor noticioso. La cuestión no es menor, en tanto quienes tienen poder $-\mathrm{y}$ recursos- para profesionalizar sus comunicaciones, podrán dominar el suministro de información (Sissons, 2012), y con ello ostentar un lugar hegemónico en el debate público.

Del otro lado de esta relación, en las redacciones, los periodistas desprecian la tarea de elaborar comunicados de prensa, desconociendo la complejidad del proceso y la relevancia 
que estos tienen para la fijación de las agendas y, finalmente, para el incremento de mayor poder simbólico de quienes están detrás.

\section{Las agencias de comunicaciones en Chile}

Hablar de control de los contenidos periodísticos por parte del poder económico hoy en Chile, es hablar de las agencias de comunicaciones o relaciones públicas y de su capacidad para influir en el quehacer periodístico. Estas han construido un creciente poder en los últimos 25 a 30 años, el cual no ha sido adecuadamente discutido o analizado, a pesar de su presencia permanente en el proceso de elaboración de noticias.

Para dimensionar esa relevancia, se puede decir que tras un fin de semana largo sin haber realizado turno, un periodista que cubre empresas puede abrir su bandeja de entrada y tener centenares de correos electrónicos pendientes enviados por alguna agencia de relaciones públicas que gestionan las estrategias comunicacionales de ciertas compañías con los medios de comunicación.

Según un conteo propio, (ver anexo) en Chile operan unas 40 de estas firmas, de distintos tamaños, focos de especialización, trayectoriay grados de éxito. Muchas de ellas son encabezadas por periodistas que dejaron el reporteo y fundaron su propia empresa en esta área, como también por ex asesores de comunicaciones de gobiernos anteriores, de políticos o incluso ex funcionarios de relaciones públicas de grandes compañías que se reciclan ofreciendo sus servicios externalizados a sus antiguos empleadores y a otros clientes que puedan conseguir. Muchas de estas empresas son filiales en Chile de transnacionales del rubro.

De acuerdo con el relato de profesionales que se desempeñan en estas empresas, algunas prestan servicios únicamente de relación de prensa, lo que incluye elaboración y envío de comunicados de prensa, organización de conferencias y convocatorias a los medios, así como la gestión de entrevistas de sus voceros y el registro de sus apariciones.

Otras son agencias de lobby más completas y gestionan reuniones privadas de ejecutivos de empresas con las autoridades públicas relevantes de su sector o consiguen la presencia de representantes políticos en las actividades que organizan las compañías y asesoran a estos ejecutivos para obtener el mejor provecho posible de tales instancias de contacto con agentes del Estado. En los últimos diez años, motivadas por la creciente demanda de Responsabilidad Social Empresarial, se ha agregado a este catálogo de servicios la relación con las comunidades aledañas a los lugares en que se espera emplazar proyectos productivos de alto impacto. Asimismo, se ofrece el manejo de crisis comunicacionales, que puede englobar todo lo anterior ante una situación adversa para la empresa.

Los clientes de estas empresas provienen de todos los rubros, aunque todas cumplen con un requisito fundamental: disponer de un presupuesto más o menos holgado para financiar este tipo de servicio. Bancos, mineras, asociaciones gremiales, supermercados, grupos financieros, fábricas de bicicletas e incluso organismos públicos las contratan. 
A la fecha, la industria de las relaciones públicas ha crecido en Chile sin regulación legal ni organizaciones que promuevan la autorregulación, como lo hace el Council of Public Relations Firms de Estados Unidos que tiene códigos voluntarios de buenas prácticas para sus 107 empresas miembro. La ley de lobby que se ingresó al Congreso en 2003, sin ser aún aprobada, no tiene por objetivo controlar la actividad de estas agencias sino que se limita a la actividad de quienes representan intereses corporativos frente a funcionarios del Estado sin mencionar la relación con los medios de comunicación.

Tal falta de regulación genera casos como que una misma agencia asesora a empresas de un determinado sector productivo o ámbito de intereses y, al mismo tiempo, hace lo propio para algún organismo de Estado que lo regula o que tiene alguna relación con él. Un ejemplo de ello es Nexos, que lista entre sus clientes al Ministerio de Vivienda y Urbanismo y al mismo tiempo a Puerto Angamos y Puerto de Mejillones, que requieren permisos emitidos por esta cartera para su operación. Otro caso es el de Hill\&Knowlton Strategies que trabaja con empresas que venden productos de consumo cotidiano como alimentos o de cuidado personal -Nestlé y Procter \& Gamble- al mismo tiempo que asesora al Servicio Nacional del Consumidor, que acoge reclamos por esta clase de artículos.

Llama la atención que hay casos en que una agencia de comunicaciones asesora a dos empresas que son competencia en sus mercados como Extend, que presta servicios a la constructora Aconcagua y a la también constructora Echeverría Izquierdo. En la mayoría de los listados también se puede apreciar que empresas que tienen relaciones de negocios potencialmente conflictivas están asesoradas por las mismas agencias de relaciones públicas, como multitiendas que arriendan espacios a centros comerciales, isapres que pagan servicios a clínicas de salud, supermercados y sus proveedores, entre otras parejas que conviven bajo el mismo techo.

La relevancia de estos conflictos de interés quedó en evidencia cuando en 2007, Hill\&Knowlton Captiva (ahora Hill\&Knowlton Strategies) terminó de manera unilateral su contrato con la empresa de televisión de pago DirecTV para asumir la gestión de comunicaciones de su competencia VTR, presumiblemente por un mayor pago. Los ejecutivos de la primera empresa hicieron ver la inconveniencia de tal situación, relevando la cantidad de información privilegiada con que contaba la agencia y que ahora podía estar en conocimiento de su competencia.

El caso Hill\&Knowlton Captiva-VTR llamó la atención sobre la profundidad de la relación entre los profesionales de estas agencias y sus clientes, de los cuales conocen sus calendarios de lanzamiento de nuevos productos y promociones, futuros nombramientos de ejecutivos, resultados financieros y del negocio, estrategias publicitarias o incluso adelantos de operaciones de fusión o de llegada y salida de un mercado.

La necesidad de regulación en las agencias de relaciones públicas es evidente. Ya sea por los conflictos de intereses que pueden tener entre sus propios clientes, los que, cuando se trata de organismo del Estado, amenazan asuntos públicos, o por la desprotección en que se encuentran los mismos clientes ante una decisión inescrupulosa de sus profesionales. 
Independiente de las características, el tamaño o la antigüedad de estas empresas o agencias de relaciones públicas, el objetivo es el mismo: ejercer lobby frente a los periodistas, en nombre de sus clientes, para controlar, dosificar o administrar la forma en que son publicadas las noticias que se refieren a sus marcas, sus gerentes, los mercados en que participan y cualquier otro aspecto que pueda ser de su interés.

\section{De "coimas" y "subsidios" de información}

Quienes conocen un poco más de cerca la mecánica de trabajo entre los periodistas y las agencias de relaciones públicas, se sorprenden de la cantidad de regalos e invitaciones que estas últimas realizan a periodistas, editores o ejecutivos de medios en el marco de sus estrategias comunicacionales. Tras la sorpresa, está la inquietud sobre los posibles efectos que tantas atenciones podrían tener en la independencia a la hora reportear y escribir noticias relativas a quienes están tras estas estrategias corporativas.

No hay estudios nacionales que permitan sostener esta sospecha: no sabemos cuánto ni de qué manera influyen los regalos en los periodistas y los contenidos que estos escriben (regalos que pueden ir desde entradas para asistir a conciertos en lugares privilegiados, presentes de Navidad, invitaciones a restaurantes exclusivos o smartphones sorteados en conferencias de prensa). Esta relación y su real incidencia en los contenidos deben ser evaluadas.

Para aproximarse a estas "coimas", Oscar H. Gandy (1982) desarrolló el concepto de "subsidios de información", definiéndolos como materiales provistos por las fuentes de manera gratuita o por debajo del costo que habitualmente pagarían por obtenerlos. Al respecto, el autor observaba que enfrentados a estrecheces de tiempo y obligados a producir historias que sean publicadas, los periodistas prestarán más atención y harán uso de ellos porque estos les van a ayudar a conseguir sus objetivos.

Los comunicados de prensa, de hecho, permiten obtener versiones de las organizaciones y sus voceros sin tener que salir del diario. El préstamo de productos costosos (como el último modelo de un automóvil o aparatos tecnológicos de alta gama) o el acceso a servicios inabordables para los periodistas (conciertos, obras de teatro, restaurantes) permiten hacer "comentarios" sin incurrir en gastos extras.

Hay dos factores que influyen en la aceptación de estas subvenciones de información. Los medios de comunicación muchas veces no tienen capacidad de producir sus propias historias y dependen más de los subsidios y, por otro lado, hay ciertos stakeholders que proveen de información a los medios y frente a los cuales los periodistas son menos desconfiados, como algunas organizaciones de la sociedad civil o las universidades (Len-Ríos et al., 2009).

Un lugar muy importante en la lista de los subsidios de información son los viajes de prensa -nacionales e internacionales-a los que invitan las agencias de relaciones públicas para llevar a los periodistas a cubrir noticias que transcurren lejos de sus lugares de trabajo, que la empresa- 
cliente quiere que reciba cobertura y que los medios -incluso los de cobertura nacional- no pueden o no quieren costear.

Inauguraciones de explotaciones mineras, represas, supermercados, puertos o carreteras, son ejemplos de actividades de relevancia periodística, más aún si cuentan con la presencia de autoridades públicas del sector y de la región. En el sentido común de los reporteros tales invitaciones no implican compromiso alguno con la empresa que organiza y paga el viaje, puesto que su valor noticioso es bastante evidente.

Si estos viajes reducen o no la independencia de los periodistas es menos relevante que constatar que estos les permiten a los grupos económicos desplegar todos sus recursos comunicacionales y discursivos, con los cuales no solo son capaces de influir en la construcción de la narración mediática, sino también pueden controlar variables de los eventos comunicativos, tales como la ocasión, el tiempo, el lugar y la presencia o ausencia de algunos participantes en tales eventos (Van Dijk, 1993).

En estos viajes y en las conferencias de prensa que incluyen no se escucha la voz de las comunidades locales que estuvieron en contra del proyecto que se está inaugurando o de los trabajadores que tienen algún reclamo y rara vez, sino nunca, se invita a medios de comunicación que contrasten los discursos de los organizadores.

Muchas de esas formas de control serían inaceptables si fueran ejercidas usando formas de fuerza física o mediante métodos violentos. Sin embargo, se aceptan sin mayor cuestionamiento. Tal legitimación surge porque estas se concretan a través de estrategias de imposición eufemizadas (Bourdieu, 1979).

Los viajes de prensa también se conectan con la segunda perspectiva propuesta, la de la formación de lazos personales con las fuentes. Cuando los traslados son para cubrir eventos de claro interés periodístico no se establece compromiso entre el periodista y la empresa, cosa que sí ocurre cuando la relevancia no es tan evidente, como en la presentación de un nuevo modelo de automóvil, la apertura de un nuevo restaurante o la oferta turística de una determinada región. En ese caso, al aceptar el viaje, los periodistas y el medio de comunicación, asumen que deben publicar algo favorable al organizador.

Sin embargo, el rédito más importante que obtiene la empresa-cliente de la invitación, es el hecho de que los periodistas que asisten tienen una vivencia común y compartida con sus ejecutivos y voceros, además de con los mismos funcionarios de las agencias. Esto también se da en cenas, conciertos o en actividades familiares de fin de semana, todas con el objetivo de generar un recuerdo que una y genere un lazo.

Más allá de una visión moral sobre la prudencia de aceptar tales invitaciones o participar en tales actividades, que apela a la debida distancia con las fuentes, estas "ofertas" deberían ser analizadas desde su funcionalidad de controlar el contenido de la prensa mediante la construcción de lazos personales de confianza y complicidad.

Con todas estas herramientas, los relacionadores públicos y los periodistas han establecido un esquema de relaciones simbióticas, en el cual las normas de dar y recibir se negocian 
continuamente (Turow, 1989). Como ya se ha dicho, las empresas-clientes y las agencias ayudan a los reporteros a resolver el mayor problema del quehacer cotidiano, que es la generación de temas para ser propuestos en reuniones de pauta, en un esquema de producción industrial de contenidos. Por cierto, aquellos que combinen mejor ubicación dentro de este esquema de relaciones con una mayor relevancia del medio de comunicación en que trabajan, obtendrán más contenidos desde estas fuentes. Entrevistas exclusivas, adelantos de información, filtración de datos y un largo etcétera son algunas de las "mercancías" que se intercambian en este "mercado".

El trabajo de las agencias a través de la construcción de lazos y de vivencias compartidas, logra su objetivo una vez que el periodista siente complicidad con la empresa-cliente y sus ejecutivos y voceros, cuando cree que hay una relación de confianza entre ambos y que sostener una actitud crítica es una traición y una decepción afectiva hacia ellos.

En relación a los subsidios de información, en la prensa chilena, vale destacar que estos son sobre todo económicos, especialmente en el caso de los viajes. A pesar de ser organizados y financiados en su totalidad por agencias de comunicaciones que nada tienen que ver con el diario, los editores los administran como incentivos para sus reporteros al momento que los reparten entre los miembros de su sección, sin considerar si la especialización del reportero se relaciona con el tema de la invitación. De esta manera se busca mantener un equilibrio y no propiciar envidias en el equipo. Incluso, los viajes se presentan en las negociaciones de contrato, argumentando que aunque el sueldo sea bajo, el puesto incluye la posibilidad de conocer otros lugares. Además de afectar la calidad del periodismo, porque, por ejemplo, un periodista de minería va a la presentación de un teléfono, estas actitudes representan una relación fiduciaria entre los medios de comunicación y las empresas de relaciones públicas.

\section{Relaciones públicas y periodismo: dos éticas en conflicto}

La frecuencia e intensidad de las interacciones entre profesionales de las relaciones públicas y los periodistas hace creer a ambos mundos que entre ellos habría una alianza estratégica, olvidando que desde el punto de vista ético de lo que ambos oficios son, en realidad están en conflicto. Incluso los textos de periodismo tienen una actitud de sospecha, pero tolerante hacia las relaciones públicas en la que se reconoce la inevitabilidad de una relación simbiótica (Turow, 1989).

Desde el punto de vista de los ideales clásicos del periodismo (independencia, servicio público, búsqueda de la verdad, libertad de expresión, pluralismo, etc.) las relaciones públicas forman un campo estructuralmente incapaz de generar una práctica ética coherente. Esto significa que incluso aquellos con buenas intenciones no son capaces de actuar éticamente sin perjudicar su capacidad de ser buenos agentes de relaciones públicas. Por el contrario, la práctica de un buen periodista está necesariamente orientada por la ética. El periodismo puede 
generar su propia buena práctica, la que es totalmente compatible con ser un profesional del periodismo (Salter, 2005.).

Primoz Krasovec e Igor Z. Zagar (2009) estudiaron las formas de organización que desarrollaron los periodistas en Eslovenia para protestar y hacer ver su posición contra la precarización de su trabajo. Uno de los problemas que encontraron los autores para abordar su objeto de estudio fue la definición de este, por lo que recurrieron al concepto de campo de Pierre Bourdieu, resumiéndolo en un agregado de relaciones sociales y prácticas que envuelven un modo común de producción social, con un discurso específico y criterios específicos de inclusión/exclusión. Los investigadores concluyeron que al periodismo solo se ajusta uno de estos criterios: que es un discurso específico.

Como se analizó antes, la producción de textos periodísticos, en cuanto a sus medios y modos no difiere de la que hace un experto en relaciones públicas, alguien que redacta invitaciones, catálogos o publicidad, lo que distingue al texto periodístico de otros textos similares no es la forma en que son producidos sino sus características específicas de género y la adherencia a cierta ética profesional. Sin embargo, esto es difícilmente suficiente para conceptualizar al periodismo como un campo en sí mismo (Krasovec et al., 2009).

La adherencia a esta ética profesional implica que un buen periodista contrastará las fuentes, observará los intereses que hay detrás de ellas, verificará los datos, procurará la mayor cantidad de puntos de vista relevantes para incluir en sus notas y en la redacción relevará información que exprese su visión del bien común. En cambio, para un buen agente de relaciones públicas implica trabajar para la representación de los intereses de su cliente buscando que dicha representación le sea rentable (o, por delegación, para la agencia de relaciones públicas). Para un agente de relaciones públicas representar a British Nuclear Fuels y tener un sentido responsable de la vida pública en las cercanías de un reactor nuclear es una especie de contradicción, ya que es la empresa y no el público el que paga (Salter, 2005).

Otro punto, quizás más problemático, es que las relaciones públicas, en contraste con el periodismo, se refieren sobre todo al mundo de las apariencias. En este sentido, para el agente de relaciones públicas, importa menos por qué o cómo se produce realmente $\mathrm{X}$ que por qué $\mathrm{o}$ cómo X parece ocurrir a los ojos de la opinión pública (Salter 2005).

Todo lo anterior no significa que las agencias de relaciones públicas y su trabajo tengan un valor menor al del periodismo, solo trae a la superficie la importancia de distinguir sus respectivas éticas, que cada uno reconozca a cuál de las dos responde y desde allí desarrollen su labor en atención a los principios que los rigen.

\section{El necesario contrapeso al poder económico}

Finalmente, es posible constatar que el creciente poder las agencias de relaciones públicas en el quehacer de los periodistas es el cada vez mayor poder del dinero en la lucha por el poder simbólico. Es una herramienta que, de acuerdo con la experiencia profesional, ha demostrado 
efectividad para controlar contenidos, capturando la capacidad crítica de los periodistas, omitiendo voces que les son inconvenientes en eventos de prensa o imponiendo su punto de vista con una publicación literal de sus comunicados.

Su desarrollo responde a condiciones históricas materiales y culturales que son difícilmente alterables, al menos en el corto plazo, por lo que lo que resta para los reporteros es reconocer esos contextos y trabajar en consecuencia con ellos y observando cuáles son los principios éticos que rigen a cada mundo profesional.

Sobre lo que se debe llamar la atención es que otros poderes no económicos, como los de las $\mathrm{ONG}$, organizaciones sociales, vecinales, sindicatos, grupos ambientales o de defensa de los derechos de los consumidores, han demostrado una incapacidad para reaccionar a estos contextos. Algunos de ellos, más lentos que otros, han olvidado desarrollar estrategias profesionales para capturar el discurso público que se construye sobre sus intereses en la prensa y no son capaces contrapesar el abrumador despliegue de recursos financieros, intelectuales, creativos, comunicacionales y discursivos de las empresas.

Casos como los de Patagonia sin Represas, el Movimiento de Integración y Liberación Homosexual y la Fundación Iguales, la organización ambientalista Oceana o la Fundación Sol, son ejemplos de instituciones conscientes de la relevancia de participar activamente en la discusión pública y del uso de subsidios de información. Estas fuentes, entre otras, están en permanente contacto con los periodistas proveyendo comunicados de prensa, ofreciendo entrevistas a voceros validados por su conocimiento de los temas, entregando datos con valor noticioso y se despliegan en las redes sociales de Internet con fuerza.

Las grandes empresas compensan la falta de legitimidad de sus argumentos mediante el uso intensivo de su capital financiero para comprar el servicio de agentes que capten la esquiva atención de las audiencias. Quisieran ellas contar con un punto de partida moral tan alto como el de una organización social que no despierta sospechas sobre sus intereses en un contexto de desconfianza y falta de credibilidad generalizada. La falta de "recursos" -dinero- no es excusa. 


\section{ANEXO}

Listado de agencias y marcas para las que prestan servicios. Esta lista no es exhaustiva, no incluye a todas las que funcionan en Chile, sino que solamente a aquellas de las que se cuenta con información verificada sobre sus clientes y que han mostrado alguna interacción con periodistas en noviembre de 2012.

\begin{tabular}{|c|c|}
\hline Agencia & $\begin{array}{l}\text { Marcas para las que prestan servicios de gestión de prensa (actualizado a } \\
\text { diciembre de 2012). }\end{array}$ \\
\hline Nexos & $\begin{array}{l}\text { Banco de Chile, Skotiabank, Asociación de Bancose Instituciones Financieras, } \\
\text { Consorcio, IM Trust, Grupo Security, AFP Cuprum, PriceWaterhouse } \\
\text { Cooper, Minera Los Pelambres, Colbún, Mina Invierno, Antofagasta } \\
\text { Minerals, GeoPark, E-CL, Enaex, Li3 Energy, SN Power, SKC, Indura, Sigdo } \\
\text { Koopers, Lipigas, Grana y Montero, Puerto Angamos, Puerto de Mejillones, } \\
\text { TGN, Fipes, SalmonChile, AGIP, Soprole, Carozzi, CCU, Aramark, Chile } \\
\text { Crece Sano, Vitamina, Inacap, Bolsa de Comercio de Santiago, Casa de } \\
\text { Moneda, Acción RSE, Ministerio de Vivienda y Urbanismo, Azul Azul, SCD. }\end{array}$ \\
\hline Extend & $\begin{array}{l}\text { Aconcagua, Aconcagua Foods, Agrosuper, Aguas Antofagasta, Aguas Nuevas, } \\
\text { Aguas del Valle, Aldeas Infantiles SOS, Aramark, Austral Capital, Australis, } \\
\text { Avon, Barrick, Chevrolet, Chile Transparente, Comsa Ente, Coopeuch, } \\
\text { Córpora, Cruz Blanca Salud, Isapre Cruz Blanca, DLP, Echeverría Izquierdo, } \\
\text { Electro Austral, Embatur, Entel, Esval, Explora, Fuerza Aérea, GE, Gersa, GM } \\
\text { Chile, Hertz, Indisa, Ingevec, Integramédica, Intersystems, Laboratorios Rider, } \\
\text { La Polar, Mall Plaza, MetLIfe, Montblanc, Parque del Recuerdo, Pizarreno, } \\
\text { Polpaico, Presto, Puerto Ventanas, Salfa Corp, Sodimac, Sociedad Nacional } \\
\text { de Minería, Supermercados de Chile, Telepizza, Tres Montes Luchetti, Tur } \\
\text { Bus, Universidad Pedro de Valdivia, Walmart Chile, Walmart Inmobiliaria. }\end{array}$ \\
\hline E-press & $\begin{array}{l}\text { ACTI, BancoEstado, BusTv, Capel, Cardumen, Carestream, Citrix, Comercial } \\
\text { Peumo, DirecTV, EMC, Fox, Google, Happyshop, IAB, Indra, Infor, Iomega } \\
\text { Chiley Perú, Kross, Kuapay, McCann, Michael Page, Oracle, Orión, Paperless, } \\
\text { Promoplan, Publiguias, Rock\&Code, Taget DDI, Universal. }\end{array}$ \\
\hline $\begin{array}{l}\text { Hill\&Knowlton } \\
\text { Strategies }\end{array}$ & $\begin{array}{l}\text { Nestlé, Procter \& Gamble, Pampers, Oral-B, Gillette, CorreosChile, Coca- } \\
\text { Cola Embonor, Puerto Valparaíso, Mc Donald's, Gasco GLP, Tucapel, PF, } \\
\text { Fasa, LG, Diageo, Baileys, Smirnoff, Pampero, Johnnie Walker, KetelOne, } \\
\text { Cacique, J\&B, Tanqueray, Ron Zacapa, Teck, MSD, Petrobras, Arcor, HSBC, } \\
\text { Remington, CGE, Transnet, Enerplus, Conafe, Emel, Edelmag, Novanet, } \\
\text { IGSA, Dreams, Sernac, Conicyt, Independencia AFI, Desafío Chile, } \\
\text { Municipalidad de Colina, Transunión, Cultivos Marinos Chiloé, Sun Edison, } \\
\text { Autodesk, Sandisk, Cameo Chile. }\end{array}$ \\
\hline Mazalán & RedHat, McAfee, Level 3 Communications, FreeLancer, Dell. \\
\hline
\end{tabular}




\begin{tabular}{|c|c|}
\hline Factor C & $\begin{array}{l}\text { Microsoft, Lenovo, Basf, Banco Interamericano de Desarrollo, Sodexo, } \\
\text { Sodexo Soluciones de Motivación, Fontaine \& Cia, Policía de Investigaciones } \\
\text { de Chile, Asociación de Empresas de Telefonía Móvil, Aguas Andina, Andess. }\end{array}$ \\
\hline IMS Márketing & Toshiba, Lexmark, Olympus, Adobe, RIM, Wacom, Totvs, Panduit, Linksys. \\
\hline Azerta & $\begin{array}{l}\text { Algeciras, Octopus LNG, Alsacia, Ami Chile, Ariztía, Aventura Center, } \\
\text { Invesa Briones, Cencosud, Costanera Center, Comité de Retail Financiero, } \\
\text { Corporación La Esperanza, Easy, Iansa, Essbio, Forus, Fundación Trascender, } \\
\text { Gener, Grupo Costanera, Hortifrut, Infanti, Johnson's, Jumbo, Lego, } \\
\text { Los Héroes, ProChile, María Ayuda, Masisa, MPX, Teatro Municipal de } \\
\text { Santiago, Paris, Recycla, Red de Alimentos, Santa Isabel, Transelec, Ultramar, } \\
\text { Schwäbisch Hall. }\end{array}$ \\
\hline ASK & $\begin{array}{l}\text { Aprocor, Arcor, Desa, Vina Errázuriz, Fox Sports, Grunenthal, IBM, Imation, } \\
\text { Kimberly Clark, Molymet, Philips, Robert Half, Ceresita, Accuhealth, TTM, } \\
\text { Molycop, UPS. }\end{array}$ \\
\hline $\begin{array}{l}\text { Bernstein \& } \\
\text { Woodward }\end{array}$ & $\begin{array}{l}\text { Proexport Colombia, Procorp, Contract, Daw, Starbucks, Laboratorios } \\
\text { Genzyme, Three Monkeys Eyewear, BYD, ZX, DSFK, Falabella Retail, } \\
\text { Motorola, DRS, Valle Nevado, Viajes Falabella, Ministerio de Justicia, } \\
\text { Seminarium. }\end{array}$ \\
\hline Meta-Group & $\begin{array}{l}\text { Bain\&Company, AMD, Avaya, Sennheiser, Norton Internet Security, } \\
\text { Symantec, }\end{array}$ \\
\hline BMR & $\begin{array}{l}\text { VMWare, Cisco, Canon, Western Digital, GE Iluminación, Triumph, Jordán } \\
\text { y Barahona Abogados. }\end{array}$ \\
\hline Presscom & $\begin{array}{l}\text { Previred, Adexus, Pragma, NCR, Netline, Gemalto, Ashley Madison, Eapro, } \\
\text { Anida. }\end{array}$ \\
\hline Alkance & $\begin{array}{l}\text { Natura, Vina Emiliana, Tetra Pak, Moure y Cía., Honda Motors, 3M } \\
\text { (Prevención), Clarke Modet \& Co. }\end{array}$ \\
\hline Feedback & Ripley, GNL Quintero, Guacolda, Chilena Consolidada, CAF. \\
\hline Burson Marsteller & $\begin{array}{l}\text { Accenture, Bayer, Ford, O2Fit, Nike, Pepsico, Danone, Autopista Central, } \\
\text { Electrolux, Black Rock, Cámara Chilena de la Construcción, Puerto San } \\
\text { Antonio, Enel Green Power, CAP, Monticello, Saxo Bank, Everis, L'Oreal, } \\
\text { Nextel, Enel, Equifax, GAES, Praxair, Caja Los Andes, Fisbury, Autopista } \\
\text { Central, Shire, Dlink, American Airlines, Intel, Sony, Symantec, Indra, Xerox, } \\
\text { Principal, Techo. }\end{array}$ \\
\hline Imaginacción & $\begin{array}{l}\text { Cruz Verde, Clínicas de Chile, SMU, CorpGroup, SQM, ADT, Claro, } \\
\text { Universidad Santo Tomás, Asociación Chilena de Seguridad. }\end{array}$ \\
\hline Headline & $\begin{array}{l}\text { Sodexo, Contructora Gardilcic, GSK, KDM, Contrutec, JOYGlobal, } \\
\text { Metecno, ME, Educamundo, Calderon y Cía, Hochtief-Tecsa, Comscore, } \\
\text { Atentus, Tecsa, Kepler, Salcedo y Cía, Aerotas, Baker y McKenzie, DBM, } \\
\text { AstraZeneca, 3E, Anabalon Espinosa, Empresas Davis, Yamana Gold, Nexo, } \\
\text { Destino Punta del Este, Groeneveld, Flores, Geocom, Coprin, Hablaitel, } \\
\text { ISA, Mundomóvil, Geovita, Muelles de Penco, Travelport, Paz Froimovich, } \\
\text { Triumph, Organon, O Boticário. }\end{array}$ \\
\hline
\end{tabular}




\begin{tabular}{|c|c|}
\hline CR Comunicaciones & $\begin{array}{l}\text { Sonda Cloud Computing, Feller Rate, Laboratorio Sanofi, Unísono, } \\
\text { Fundación Fueyo-UDP, Previsionarte. }\end{array}$ \\
\hline Neurona-Group & $\begin{array}{l}\text { Edutic, Softland, Clínica Cordillera, Clínica Los Carrera, Salva la Tierra, } \\
\text { Tecnociencia. }\end{array}$ \\
\hline Contacto 21 & $\begin{array}{l}\text { Departamento de Cirugía Vascular y Endovascular de la Universidad } \\
\text { Católica, Universidad Mayor, Royal Canin, Nintendo, Samsung, Kingston, } \\
\text { APC, Polycom, Jarden Corporation, Capital ED }\end{array}$ \\
\hline Ko2 & $\begin{array}{l}\text { Help, PetroMagallanes, Chilevisión, Banmédica, Clínica Vespucio, Vida } \\
\text { Tres, Sonda, Derco, Clínica Ciudad del Mar, Avina, Coca-Cola de Chile, } \\
\text { Mc Donald's, Fundación Banmédica, Mondolez, Clínica Santa María, Vida } \\
\text { Integra, Clínica Dávila. }\end{array}$ \\
\hline Tironi y Asociados & $\begin{array}{l}\text { Anber, Sura, AFP Capital, Morfo, Petrobras, Movistar, Banco Santander, } \\
\text { Embotelladora Andina, Unilever, Enersis, Chilectra, Angloamerican, } \\
\text { Syngenta, Transbank, RSA, Consejo Minero, Consalud. }\end{array}$ \\
\hline Generacom & World Economic Forum, Ericsson, 4G Americas, Sony Mobile. \\
\hline Quorum & $\begin{array}{l}\text { Marbella, La Serena Golf, Marina Golf Rapel, San Alfonso del Mar, Ralei, } \\
\text { RVC, Manquehue - Piedra Roja, Fiat, Alfa Romeo, Maserati, Ferrari, Jeep, } \\
\text { Dodge, Chrysler, RAM, Bulova, Casio, Timex, Cacharel, Náutica, Asociación } \\
\text { de Aseguradoras de Chile. }\end{array}$ \\
\hline Quásar & $\begin{array}{l}\text { Empresas Copec, Abastible, Nvidia, Sonamar, Sonacol, Bosch, Qualcomm, } \\
\text { Arauco, Terpel, Orizon, ANA, Numancia, Vivo Positivo, Arco Prime, Golden } \\
\text { Omega, Mobil. }\end{array}$ \\
\hline Vértices & $\begin{array}{l}\text { Apple, Drag Pharma, Saxoline, Pretty, Womane, Vitta Oral, Les Gemeaux } \\
\text { Esthetique. }\end{array}$ \\
\hline Urban & Nokia, Unilever, Sedal, Dove, Tresseme, Clear, Nars, Rapsodia, Prune. \\
\hline Dos Alas & $\begin{array}{l}\text { Cencosud, Hertz, La Crianza, Indumotora, BNP Paribas, Viajes Falabella, } \\
\text { La Roche-Posay, Motorola, Carrera, Colcci, Columbia, Grey Góose, } \\
\text { Grupo Belén, Heineken, House, Kia, Mistral, Vina Morandé, Puma, Royal } \\
\text { Caribbean, Viajes Falabella, Virgin Mobile, Ash, Bacardi, BNP Paribas Cardif, } \\
\text { Burn, Carolina Herrera, Converse, Diesel, Digital Bond, Bauzá, Echinuco, } \\
\text { Elite, Eristoff, Gacel, Gama Professional, Interexpress, Italmod, Lippi, Johnnie } \\
\text { Walker, Levi's, Lamography, Loud, Martini, Mia, Mistral, Nestlé, Opaline, } \\
\text { Zacapa, Feria del Sanguche, Steve Madden, Svelty, Tierra Atacama, Tommy } \\
\text { Hilfigher, Trek, UFO, Umbrale, Bar Constitución. }\end{array}$ \\
\hline Vox Comunicaciones & $\begin{array}{l}\text { AbcDin, AlbagliZaliaznik, Arrigoni, AutoGasco, BancoEstado Pequenas } \\
\text { Empresas, Bobble, Chile Arte Contemporáneo, Claro y Asociados, Clínica } \\
\text { Alemana, Clínica Las Lilas, Edmond de Rothschild, Energy Partners, Era, } \\
\text { Fraunhofer, Fundación Cristo Vive, Gourmet, Havas Media, Ideal, Instore } \\
\text { Media Group, Integration, Kaufmann, Lan, Masvida, Metrogas, Nexans, } \\
\text { Marubeni, Philip Morris, Rotter \& Krauss, Siemens, Sociedad Chilena del } \\
\text { Litio, Tecno Fast Atco, Universidad de Los Andes, xDirect. }\end{array}$ \\
\hline
\end{tabular}




\begin{tabular}{|c|c|}
\hline Inter-Medios & $\begin{array}{l}\text { Faw, Michelin, Newll Rubbermaid, Mabe, Vina Montes, Props Chile, A10 } \\
\text { Networks, Torre Colón, Droguería Hoffmann, Fabrics, Seniority. }\end{array}$ \\
\hline Fecunda & $\begin{array}{l}\text { BMW, Rolls Royce, Hasbro, Prosegur, Multitiendas Corona, JIS Group, } \\
\text { Dafiti, Fundación Nuestros Hijos, Fundación Copec-UC, Asociación de } \\
\text { Laboratorios Farmacéuticos, Escuela de Posgrados de la Facultad de Artes de } \\
\text { la Universidad de Chile, Mi Espacio Siestario, Programa de Apoyo a Víctimas } \\
\text { del Ministerio del Interior y Seguridad Pública. }\end{array}$ \\
\hline $\begin{array}{l}\text { Proyecta } \\
\text { Comunicaciones }\end{array}$ & $\begin{array}{l}\text { Finnning-Cat, AquaChile, Moneda Asset Management, Cruz del Sur } \\
\text { Servicios Financieros, Thesis Consultores, BCG, Codelco, Chilexpress, } \\
\text { CTPartners, Froimovich, BSA, Marsh. }\end{array}$ \\
\hline Communicarte & $\begin{array}{l}\text { Swarovski, Dimacofi, Skechers, StreetMachine, Comética, Amanoz, Intex, } \\
\text { Infanti, Silfa, Avent, Chicco, Pao, Bodum, Pyrex, Cuisinart, Lego, Chicago } \\
\text { Cutlery, El Colorado, Luxaflex, MGM, Agua, Puig, Vendetta, Corporación } \\
\text { Participa, Fabrics Home, Casa Tv, Mestizo, Trotter, ISJ, LA Gear. }\end{array}$ \\
\hline MG Consulting & $\begin{array}{l}\text { Alaluf Propiedades, Falcom MP, Bright Star (Cucci, Lacoste, Escada, } \\
\text { Dolce\&Gabbana, Puma, Hugo Boss, Murad, Sisley, Paris, Senscience.), } \\
\text { Novared, Swarosvki, La Fete Chocolat, Atrápalo, Bermudez Mora Abogados, } \\
\text { Chilextremo, Kaufmann, CSAV, Luxury. }\end{array}$ \\
\hline Alianza Com & $\begin{array}{l}\text { Kunza, BancoEstado, Ultravisión, Munchi's, San Isidro Labrador, Asociación } \\
\text { de Empresas de Energía Solar, Forja Chile, Trend Management, Farenhouse, } \\
\text { Puntaje Nacional, North Land, FeriaTicket, Asiamerica. }\end{array}$ \\
\hline Ogilvy & $\begin{array}{l}\text { Nestlé (Milo, Chiquitín, Chandelle, Svelty, Chamyto, Yoghurt, Postres), } \\
\text { Jumbo (Producto Jumbo, Jumbo Pack, Jumbo te da más), Cervcerías Chile } \\
\text { (Becker, Brahma, Stella Artois, Baltica, Malta del Sur, Beck's), Unilever } \\
\text { (Helmann's, Ades, Té Club, Le Sancy, Dove, Pond's, Soft, Huggies, Kotex, } \\
\text { Poise, Plenitud), AFP Cuprum, Bubble Gummers, Barbie, Unicef, Balthus. }\end{array}$ \\
\hline Creaciones Panisello & $\begin{array}{l}\text { Isapres, AEF, Contémpora, Copeval, Recipet/Typack, Coppelia, Preunic, } \\
\text { Pritt, Copa Airlines, Dunkin Donuts, Soda Stream, Selray, Dominó Fuente de } \\
\text { Soda, Loctite, Constanini Joyas, Inmobiliaria y Constructora Vimac, Tierra } \\
\text { Noble, Nestlé, Agorex, Henkel, Hyundai, Grupo Gildemeister. }\end{array}$ \\
\hline Rumbo Cierto & $\begin{array}{l}\text { Munita, Cruzat y Claro; Global Folio; AFP Cuprum; Banco Penta; Vantrust } \\
\text { Capital; Mapcity; Credit Suisse; Penta Security. }\end{array}$ \\
\hline
\end{tabular}




\section{Referencias bibliográficas}

Bravard, J. y Morgan R. (2007). La externalización inteligente. Barcelona: Deusto.

Bourdieu, P. (1979). Symbolic Power. Critique of Anthropology, 4, 77-85.

Davies, A. (2000). Public relations, business news and the reproduction of corporate elite power. Journalism, 1, 282-304.

Davies, A. (2000). Public relations, news production and changing patterns of source access in the British national media. Media Culture Society, 22, 39-59.

Edwards, L. (2012). Defining the 'object' of public relations research: A new starting point. Public Relations Inquiry, 1, 7-30.

Edwards, L. (2006). Rethinking power in public relations. Public Relations Review, 32, 229231.

Gandy, O. (1982). Beyond Agenda Setting: Informations subsidies and public policy, Ablex. New Jersey: Norwood.

Krasovec, P. y Zagar, I. (2009). Divisions and struggles of the Slovenian journalistic guild. Journalism Studies, 10 (1), 85-99.

Larraín, G. (2005). Chile, fértil provincia: hacia un estado liberador y un mercado revolucionario. Santiago: Debate.

Len-Rios, M., Hinnanf, A., Park, S., Cameron, G., Frisby, C. y Lee, Y. (2009). Health News Agenda Building: Journalists' Perceptions of the Role of Public Relation. Journalism \& Mass Communication Quarterly, 85, 315-331.

L'Etang, J. (2004). Public Relations in Britain: a History of Professional Practice in the Twentieth Century. Mahwah, New Jersey: Lawrence Erlbaum Associates.

Salter, L. (2005). The communicative structures of journalism and public relations. Journalism, 6(1), 90-106.

Sissons, H. (2012). Journalism and public relations: A tale of two discourses. Discourse \& Communication, 6, 273-294.

Turow, J. (1989). Public Relations and Newswork: A Neglected Relationship. American Behavioral Scientist, 33, 206-212.

Sunkel, G. y Otano, R. (2003). Libertad de los periodistas en los medios. Comunicación y Medios, 14. Obtenido el 14 de diciembre de 2012, desde http://www.icei.uchile.cl/ portal/revista-comunicacion-y-medios/49541/n14-ano-2003 .

Van Dijk, T. (1993). Principles of Critical Discourse Analysis. Discourse Society, 4, 249-283. 